\title{
The Boltzmann Equations in General Relativity
}

\author{
By A. G. Walker, University of Oxford.
}

(Received 2nd November, 1935. Read 6th December, 1935.)

\section{§1. Introduction.}

In a recent paper ${ }^{1}$, J. L. Synge gives an interesting derivation of the conservation equations $T^{i j}{ }_{j}=0$ satisfied by the energy tensor $T^{i j}$ of a continuous medium. Previous to the appearance of this paper, these equations were generally obtained by assuming the classical equations of motion and continuity, after which it was necessary to appeal to the Principle of Equivalence ${ }^{2}$. It then follows that the path of a free particle is a geodesic. Synge however starts with the hypothesis that the path of a particle between collisions is a geodesic and that the proper mass is constant. The conservation equations are then deduced exactly from the law of conservation of momentum for collisions.

The object of the present paper is to consider the particular case of Synge's problem that arises when it is assumed that no collisions take place. In this case, conservation of momentum can be replaced by conservation of particle number, i.e. a system of particles is required to satisfy the condition that no particle shall be created or destroyed. Thus if we choose in space-time a three-dimensional surface which is such that the world-line of any particle will meet this surface once and once only, then since the world-lines are prescribed, the distribution of particles at any point of space-time can be determined when the distribution at each point of this surface is known. Hence if the system is specified by distribution functions (one for material particles and another for photons) at each point of space-time, these functions are restricted in that they must satisfy certain differential equations, the generalised Boltzmann equations. The boundary conditions which determine particular solutions of these equations are given by the forms of the distribution functions

\footnotetext{
1 Trans. Roy. Soc., Canada (3), 28 (1934), 127-171.

2 See, for example, Eddington, "Mathematical Theory of Relativity," $\$ 53,54$.
} 
at points of the surface mentioned above. The main object of this paper is to obtain the Boltzmann equations for general space-time. No general solution of these equations has yet been found, but it is seen that the problem of finding a solution is exactly the problem of finding a first integral of the geodesic equations.

When the distribution of particles is known, the energy tensor for the system can be found, and it is verified as a matter of interest that the energy tensor satisfies the conservation equations $T^{i j},{ }_{j}=0$ as a consequence of the Boltzmann equations. This is to be expected, for Synge has derived these equations in the more general case when collisions are admitted.

In the general theory of relativity, the energy tensor is identified with a certain geometrical tensor, so that the macroscopic distribution can be determined when the form of space-time is given. The geometry does not however determine explicitly the microscopic distribution, and it is evident that the problem of finding this distribution is insoluble if collisions are admitted. The problem is more precise if collisions are neglected, although even then we should not expect the solution to be unique; it is possible however that one distribution is to be preferred to others on grounds of symmetry, etc. This converse problem is of some interest from the geometrical, if not physical, point of view. No general solution has yet been obtained, but a particular case is considered in the last section of this paper.

We deal with a continuous distribution of particles, and it is therefore necessary to consider separately the distribution on the velocity boundary, i.e. the distribution of photons. This is permissible, for in the absence of collisions material particles and photons evidently constitute systems satisfying separately the law of conservation of number. It follows that the energy tensor will be the sum of two parts, one being provided by material particles and the other by photons.

\section{§2. Material particles.}

The hypothesis concerning material particles is that a particle is free from collisions, its proper mass is constant, and its path is a geodesic in space-time. If the metric of space-time is

$$
d s^{2}=g_{i j} d x^{i} d x^{j}
$$

where $i, j$ take the values $0,1,2,3$, the unit vector tangent to the 
path of a particle is $\lambda^{i}=d x^{i} / d s$. This vector is time-like so that, adopting the usual convention ${ }^{1}$ that the indicators of time-like and space-like vectors are positive and negative respectively, the components must satisfy the relation

$$
g_{i j} \lambda^{i} \lambda^{j}=1 \text {. }
$$

Also since the path is a geodesic,

$$
d \lambda^{i}+\Gamma_{j k}^{i} \lambda^{j} d x^{k}=\mathbf{0}
$$

where $\Gamma_{j k}^{i}$ are the Christoffel symbols of the second kind. It is assumed that the arc $s$ increases in the direction of propagation along a path, so that the vector $\lambda^{i}$ points from the past to the future. This will be assumed for all vectors $\lambda^{i}$ mentioned in this paper.

The momentum vector associated with a particle of proper mass $m_{0}$ is

$$
p^{i}=m_{0} \lambda^{i} .
$$

This vector therefore has the sense of $\lambda^{i}$, and since $m_{0}$ is constant by hypothesis, then from (2) and (3), the components satisfy

$$
\begin{gathered}
g_{i j} p^{i} p^{j}=m_{0}^{2} \\
d p^{i}+\Gamma_{j k}^{i} p^{j} d x^{k}=0 .
\end{gathered}
$$

From special relativity, this momentum vector has the property that at an event $P$, the energy of the particle relative to an observer at $P$ is

$$
E=c^{2}\left(g_{i j} h^{i} p^{j}\right)_{P}
$$

where $h^{i}$ is the unit vector tangent to the observer's world-line. For an observer moving with the particle at $P, h^{i}=\lambda^{i}$, and the energy is $m_{0} c^{2}$, from (2) and (4).

\section{§3. Distribution of material particles.}

At a point $P(x)$, let $d \Omega_{1}$ be the element ${ }^{2}$ of three-dimensional solid angle determined by the range of directions $\left(\lambda^{i}, \lambda^{i}+d \lambda^{i}\right)$. We define this solid angle as follows: Consider the flat space $E_{P}$ tangent to space-time at $P$. The metric of $E_{P}$ is $d s^{2}=\left(g_{i j}\right)_{P} d X^{i} d X^{j}$, and if $X^{i}=r \lambda^{i}$, where $\lambda^{i}$ is a unit vector at $P$, then the $X^{\prime}$ 's may be considered

1 With this convention, the signature of the quadratic (1) is -2 .

2 The subscript 1 attached to $d \Omega$ indicates that we are dealing with material particles. The subscript 2 will occur when we discuss photons. 
as the coordinates of a point $Q$ distant $r$ from $P$ in $E_{P}$. The element of volume of $E_{P}$ at $Q$ is $\sqrt{ }(-g)_{P} d X^{0} d X^{1} d X^{2} d X^{3}$, and the element of solid angle $d \Omega_{1}$ at $P$ is defined to be such that this element of volume is $r^{3} d r d \Omega_{1}$. Hence the element of solid angle corresponding to the range $\left(\lambda^{i}, \lambda^{i}+d \lambda^{i}\right)$ of unit vectors at $P$ is given by

$$
r^{3} d r d \Omega_{1}=\sqrt{ }(-g)_{P} d X^{0} d X^{1} d X^{2} d X^{3}, \quad X^{i}=r \lambda^{i} .
$$

Equation (9) below is obtained by writing $m_{0}$ for $r$ and $p^{i}$ for $X^{i}$ in this relation.

Now let $d V$ be the element of volume of a three-dimensional space orthogonal to the vector $\lambda^{i}$ at $P$. Suppose that the number of particles crossing $d V$, whose paths issue in the angle $\vec{d} \Omega_{1}$ and whose proper masses are in the range $\left(m_{0}, m_{0}+d m_{0}\right)$, is $d N$. Then this number can be expressed in the form

$$
d N=\chi^{*}\left(x, m_{0}, \lambda\right) m_{0}^{-1} d m_{0} d V d \Omega_{1},
$$

where $\chi^{*}\left(x, m_{0}, \lambda\right)$ is some distribution function. Since $m_{0}, \lambda^{i}$ can be expressed in terms of $p^{i}$ by means of (4) and (5), we can write

$$
\chi^{*}\left(x, m_{0}, \lambda\right)=\chi(x, p)
$$

Also, from (4), we have

$$
(-g)^{\frac{1}{2}} d p^{0} d p^{1} d p^{2} d p^{3}=m_{0}^{3} d m_{0} d \Omega_{1}
$$

Hence, (7) can be written

$$
d N=\chi(x, p) m_{0}^{-4}(-g)^{\frac{1}{2}} d V d p^{0} \ldots d p^{3} .
$$

The function $\chi(x, p)$ is not arbitrary, for it is required that the number of particles shall be conserved. Hence, $\chi(x, p)$ must be such that

$$
\Delta(d N)=0
$$

where $\Delta$ denotes the increment for a displacement from $P(x)$ along the path in direction $\lambda^{i}$.

We can, without loss of generality, assume that the coordinates are chosen so that the surfaces $x^{0}=$ constant are space-like, i.e. the normals to these surfaces are time-like, and that the value of $x^{0}$ increases from past to future. Then $g_{00}>0$, and the quadratic ${ }^{1}$ $g_{\mu \nu} X^{\mu} X^{\nu}$ is negative definite. Also, the vector $\lambda^{i}$ has the correct

It will be understood that Latin suffixes $h, i, j, l, l$ take the values $0,1,2,3$, and Greek suffixes $\mu, \nu$ take the values $1,2,3$. 
sense if and only if $\lambda^{0}>0$, for $\lambda^{0}=d x^{0} / d s$, and since $d s>0$, we have $d x^{0}>0$ if $\lambda^{0}>0$ and conversely.

The element of volume of a surface $x^{0}=$ constant is, from (1),

$$
d W=\left(-\left|g_{\mu \nu}\right|\right)^{\frac{1}{2}} d x^{1} d x^{2} d x^{3}=\left(-g g^{00}\right)^{\frac{1}{2}} d x^{1} d x^{2} d x^{3},
$$

and the covariant components of the vector normal to this surface are $\left(\left(g^{00}\right)^{-t}, 0,0,0\right)$. Hence, the element of volume $d V$ is

$$
d V=\lambda^{0}\left(g^{00}\right)^{-\frac{1}{2}} d W=(-g)^{\frac{1}{2}} \lambda^{0} d x^{1} d x^{2} d x^{3},
$$

and writing $p^{0} / m_{0}$ for $\lambda^{0},(10)$ becomes

$$
d N=\chi(x, p) m_{0}^{-5}(-g) p^{0} d x^{1} \ldots d p^{0} \ldots d p^{3} .
$$

Passing along the path in direction $\lambda^{i}$ from the surface $x^{0}$ to the surface $x^{0}+\Delta x^{0}$, where $\Delta x^{0}$ is small and positive, and writing $x^{i}=x^{i}+\Delta x^{i}, p^{i}=p^{i}+\Delta p^{i}$, we have from (4) and (6),

$$
\begin{aligned}
x^{i} & =x^{i}+\epsilon p^{i}, \\
p^{\prime} i & =p^{i}-\epsilon \Gamma_{j k}^{i} p^{j} p^{k}, \\
\epsilon & =\Delta x^{0} / p^{0} .
\end{aligned}
$$

where

Hence, since $\epsilon$ is small,

$$
\frac{\partial\left(x^{\prime 1} \ldots p^{\prime 0} \cdots p^{3}\right)}{\partial\left(x^{1} \ldots p^{0} \ldots p^{3}\right)}=1-\frac{\partial}{\partial p^{i}}\left(\epsilon \Gamma_{j k}^{i} p^{j} p^{k}\right),
$$

whence we find

$$
\Delta\left\langle d x^{1} \ldots d p^{0} \ldots d p^{3}\right\rangle=\epsilon\left\{-2 \Gamma_{i k}^{i} p^{k}+\left(p^{0}\right)^{-1} \Gamma_{j k}^{0} p^{j} p^{k}\right\} d x^{1} \ldots d p^{0} \ldots d p^{3} .
$$

Also, from (13) and (14),

Hence we have

$$
\begin{aligned}
\Delta(-g) & =-2 \epsilon g \Gamma_{i k}^{i} p^{k}, \\
\Delta\left(p^{0}\right) & =-\epsilon \Gamma_{j k}^{0} p^{j} p^{k} .
\end{aligned}
$$

$$
\Delta\left(-g p^{0} d x^{1} \ldots d p^{0} \ldots d p^{3}\right)=0 .
$$

Since $m_{0}$ is constant along a path, $\Delta\left(m_{0}\right)=0$, so that from (11), (12) and (15), we see that $\chi(x, p)$ must satisfy $\Delta \chi=0$, i.e.

$$
\frac{\partial \chi}{\partial x^{i}} p^{i}-\frac{\partial \chi}{\partial p^{i}} \Gamma_{j k}^{i} p^{j} p^{k}=0
$$

This therefore is the generalised Boltzmann equation that must be satisfied by the distribution function $\chi(x, p)$ in order that number shall be conserved. It can be verified that the form of this equation is invariant under transformations of the coordinates. 
Returning to the identity (8), we have from (4)

$$
\frac{\partial \chi^{*}}{\partial x^{i}}=\frac{\partial \chi}{\delta x^{i}}, \quad \frac{\partial \chi^{*}}{\partial \lambda^{i}}=m_{0} \frac{\partial \chi}{\partial p^{i}}
$$

Hence, (16) is equivalent to the equation

$$
\frac{\partial \chi^{*}}{\partial x^{i}} \lambda^{i}-\frac{\partial \chi^{*}}{\partial \lambda^{i}} \Gamma_{j k}^{i} \lambda^{j} \lambda^{k}=0
$$

§4. The energy-tensor for the material system.

The energy-tensor at a point $P$ is defined in terms of the distribution by

$$
T_{1}^{i j}=\int \lambda^{i} \lambda^{j} m_{0} d \sigma
$$

where $d \sigma$ is the proper number-density of particles at $P$, the integration being over all proper masses and all directions. From (7), the number-density is given by

$$
d \sigma=\frac{d N}{d V}=\chi^{*}\left(x, m_{0}, \lambda\right) m_{0}^{-1} d m_{0} d \Omega_{1},
$$

whence

Writing

$$
T_{1}^{i j}=\int \chi^{*}\left(x, m_{0}, \lambda\right) \lambda^{i} \lambda^{j} d m_{0} d \Omega_{1}
$$

$$
\phi(x, \lambda)=\int_{0}^{\infty} \chi^{*}\left(x, m_{0}, \lambda\right) d m_{0}
$$

the expression for $T_{1}^{i j}$ becomes

$$
T_{1}^{i j}=\int \phi(x, \lambda) \lambda^{i} \lambda^{j} d \Omega_{1} .
$$

It is easily seen from (17) and (19) that $\phi(x, \lambda)$ satisfies a similar equation, i.e

$$
\frac{\partial \phi}{\partial x^{i}} \lambda^{i}-\frac{\partial \phi}{\partial \lambda^{i}} \Gamma_{j k}^{i} \lambda^{j} \lambda^{k}=0
$$

An expression similar to (20) is obtained if it is assumed that all material particles have the same proper mass $m_{0}$. In this case, the particle number is

$$
d N=\phi(x, \lambda) m_{0}^{-1} d V d \Omega_{1},
$$

where $\phi$ satisfies (21), and the energy-tensor is found to be given by (20). It appears therefore that, for simplicity, we can assume that 
all material particles have the same proper mass. The integration giving $\phi(x, \lambda)$ from $\chi^{*}\left(x, m_{0}, \lambda\right)$ is simply equivalent to the process of combining into one particle all particles moving together.

We observe that equation (21) expresses the condition that $\phi(x, d x / d s)=$ constant shall be an integral of the geodesic equations (3). The problem of finding these integrals has been considered, but no solution for a general space has yet been obtained.

In order to obtain an explicit expression for the integral in (20), it is convenient to choose a coordinate system as in $\S 3$, and to allow $\lambda^{1}, \lambda^{2}, \lambda^{3}$ to be the independent components of the vector $\lambda^{i}$. The component $\lambda^{0}$ must now be expressed in terms of $\lambda^{\mu}$ by means of the relation

$$
g_{i j} \lambda^{i} \lambda^{j} \equiv g_{00}\left(\lambda^{0}\right)^{2}+2 g_{0 \mu} \lambda^{\mu} \lambda^{0}+g_{\mu \nu} \lambda^{\mu} \lambda^{\nu}=1 .
$$

It has been shown that the vector $\lambda^{i}$ has the correct sense if and only if $\lambda^{0}>0$. Since however $g_{00}>0$ and $g_{\mu \nu} \lambda^{\mu} \lambda^{\nu}<0$ for all real components $\lambda^{\mu}$, equation (22) for $\lambda^{0}$ has just one positive root for any values of $\lambda^{\mu}$. Hence the components $\lambda^{\mu}$ are not restricted, and the corresponding value of $\lambda^{0}$ is unique.

To find $d \Omega_{1}$, we use the relation $p^{i}=m_{0} \lambda^{i}$. We have

from (22), and

$$
\begin{aligned}
& \frac{\partial p^{i}}{\partial m_{0}}=\lambda^{i}, \\
& \frac{\partial p^{0}}{\partial \lambda^{\mu}}=m_{0} \frac{\partial \lambda^{0}}{\partial \lambda^{\mu}}=-\frac{m_{0}}{\lambda_{0}} \lambda_{\mu}
\end{aligned}
$$

It follows that

$$
\frac{\partial \boldsymbol{p}^{\nu}}{\partial \lambda^{\mu}}=m_{0} \delta_{\mu}^{\nu}
$$

whence

$$
\frac{\partial\left(p^{0}, p^{1}, p^{2}, p^{3}\right)}{\partial\left(m_{0}, \lambda^{1}, \lambda^{2}, \lambda^{3}\right)}=\frac{m_{0}^{3}}{\lambda_{0}},
$$

Hence, from (9),

$$
d p^{0} \ldots d p^{3}=\left(\lambda_{0}\right)^{-1} m_{0}^{3} d m_{0} d \lambda^{1} d \lambda^{2} d \lambda^{3} .
$$

Substituting in (20),

$$
d \Omega_{1}=(-g)^{\frac{1}{2}}\left(\lambda_{0}\right)^{-1} d \lambda^{1} d \lambda^{2} d \lambda^{3} .
$$

$$
T_{1}^{i j}=\iiint \phi(x, \lambda) \lambda^{i} \lambda^{j}(-g)^{\frac{1}{1}}\left(\lambda_{0}\right)^{-1} d \lambda^{1} d \lambda^{2} d \lambda^{3},
$$

where $\lambda^{0}, \lambda_{0}$ are to be expressed in terms of $\lambda^{\mu}$. Since the variables $\lambda^{\mu}$ are not restricted, the limits of integration are $(-\infty,+\infty)$ for each. 
§5. The conservation equations for $T_{1}^{i j}$.

It will now be proved that the tensor defined above satisfies the equations $T_{1}^{i j}, j=0$ when $\phi(x, \lambda)$ satisfies (21). Consider the value $T^{\prime i j}$ of the tensor at the point $x^{\prime i}=x^{i}+\epsilon^{i}$, where the $\epsilon$ 's are small arbitrary constants. Then from (24),

$$
T_{1}^{\prime i j}=\iiint \phi\left(x^{\prime}, \lambda^{\prime}\right) \lambda^{\prime i} \lambda^{\prime j}\left(-g^{\prime}\right)^{l}\left(\lambda_{0}^{\prime}\right)^{-1} d \lambda^{\prime 1} d \lambda^{\prime 2} d \lambda^{\prime 3},
$$

where $\lambda^{\prime 0}, \lambda_{0}^{\prime}$ are given by $g_{i j}^{\prime} \lambda^{\prime i} \lambda^{\prime j}=1, \lambda^{\prime 0}>0, \lambda_{0}^{\prime}=g_{0 i}^{\prime} \lambda^{\prime i}$. Now transform from $\lambda^{\prime \mu}$ to new variables $\lambda^{\mu}$ by means of the relations

$$
\lambda^{i}=\lambda^{i}-\Gamma_{j k}^{i} \lambda^{j} \epsilon^{k}
$$

where the $\Gamma$ 's are evaluated at the point $(x)$. It is easily verified that

$$
g_{i j} \lambda^{i} \lambda^{j}=g_{i j}^{\prime} \lambda^{\prime i} \lambda^{j}=1
$$

and that $\lambda^{0}>0$. Hence, $\lambda^{0}$ is given correctly in terms of $\lambda^{\mu}$ by (22). Further, the limits of integration are evidently unaltered.

To prove that $d \Omega^{\prime}{ }_{1}=d \Omega_{1}$, we have, from (26) and (22),

Hence,

$$
\begin{aligned}
\frac{\partial \lambda^{\prime \mu}}{\partial \lambda^{\nu}} & =\delta_{\nu}^{\mu}-\left(\Gamma_{\nu k}^{\mu}+\Gamma_{0 k}^{\mu} \frac{\partial \lambda^{0}}{\partial \lambda^{\nu}}\right) \epsilon^{k} \\
& =\delta_{\nu}^{\mu}-\left(\Gamma_{\nu k}^{\mu}-\left(\lambda_{0}\right)^{-1} \lambda_{\nu} \Gamma_{0 k}^{\mu}\right) \epsilon^{k} .
\end{aligned}
$$

Also,

$$
\begin{aligned}
\frac{\partial\left(\lambda^{\prime 1}, \lambda^{\prime 2}, \lambda^{\prime 3}\right)}{\partial\left(\lambda^{1}, \lambda^{2}, \lambda^{3}\right)} & =1-\left(\Gamma_{\mu k}^{\mu}-\left(\lambda_{0}\right)^{-1} \lambda_{\mu} \Gamma_{0 k}^{\mu}\right) \epsilon^{k} \\
& =1-\left(\Gamma_{i k}^{i}-\left(\lambda_{0}\right)^{-1} \lambda_{i} \Gamma_{0 k}^{i}\right) \epsilon^{k} .
\end{aligned}
$$

and

$$
\left(-g^{\prime}\right)^{1}=(-g)^{\ddagger}\left(1+\Gamma_{i k}^{i} \epsilon^{k}\right) \text {, }
$$

$$
\lambda_{0}^{\prime}=\lambda_{0}-\lambda_{i} \Gamma_{0 k}^{i} \epsilon^{k} .
$$

Combining these expressions, we have

$$
\left(-g^{\prime}\right)^{\frac{1}{2}}\left(\lambda_{0}^{\prime}\right)^{-1} d \lambda^{\prime 1} d \lambda^{\prime 2} d \lambda^{\prime 3}=(-g)^{\frac{1}{2}}\left(\lambda_{0}\right)^{-1} d \lambda^{1} d \lambda^{2} d \lambda^{3}=d \Omega_{1} .
$$

Thus (25) can be written

where

$$
T_{1}^{\prime i j}=\int \phi\left(x^{\prime}, \lambda^{\prime}\right) \lambda^{\prime i} \lambda^{\prime j} d \Omega_{1}
$$

$$
x^{i}=x^{i}+\epsilon^{i}, \quad \lambda^{\prime i}=\lambda^{i}-\Gamma_{j k}^{i} \lambda^{j} \epsilon^{k} .
$$


Expanding both sides of this equation and substituting from (20), we have

$$
\frac{\partial T_{1}^{i j}}{\partial x^{k}} \epsilon^{k}=\epsilon^{k} \int\left(\frac{\partial \phi}{\partial x^{k}}-\frac{\partial \phi}{\partial \lambda^{l}} \Gamma_{h k}^{l} \lambda^{h}\right) \lambda^{i} \lambda^{j} d \Omega_{1}-T^{h j} \Gamma_{h k}^{i} \epsilon^{k}-T^{i h} \Gamma_{h k}^{j} \epsilon^{k} .
$$

Hence, since these equations hold for arbitrary values of the $\epsilon$ 's,

$$
T_{1}^{i j},_{k}=\int\left(\frac{\partial \phi}{\partial x^{k}}-\frac{\partial \phi}{\partial \lambda^{l}} \Gamma_{h k}^{l} \lambda^{h}\right) \lambda^{i} \lambda^{j} d \Omega_{1} .
$$

Contracting for $j$ and $k$, we see at once that if $\phi$ satisfies (21),

$$
T_{1}^{i j},{ }_{j}=0 \text {. }
$$

Thus, if the free paths are geodesics, the energy tensor for a conserved system of material particles satisfies the equations $T^{i j}{ }_{j}=0$.

Geometrically, it has been proved that the divergence of a tensor defined by an expression of the form (20) vanishes if $\phi(x, d x / d s)=$ constant is an integral of the geodesic equations. Thus the problem of finding a second order tensor whose divergence vanishes can be reduced to the problem of finding an integral of the geodesic equations.

\section{§6. Photons.}

The hypothesis concerning photons is that a photon is free from collisions, its path is a null geodesic, and the associated momentum vector is tangent to, and propagated by parallel transport along, the path. Thus the momentum vector is given by $p^{i}=d x^{i} / d \tau$, where $\tau$ is a suitable parameter along the path, and the components satisfy the equations

$$
\begin{gathered}
g_{i j} p^{i} p^{j}=0 \\
d p^{i}+\Gamma_{j k}^{i} p^{j} d x^{k}=0
\end{gathered}
$$

From the definition of this vector, it points from the past to the future, and at an event $P$, the energy of the photon relative to an observer at $P$ is

$$
E=c^{2}\left(g_{i j} h^{i} p^{j}\right)_{P},
$$

where $h^{i}$ is the unit vector tangent to the observer's world-line.

1 The vector $p^{i}$ will refer to photons in the remainder of this paper, and should not be confused with the momentum vector associated with a material particle. 


\section{$\S 7$. The distribution of photons.}

Since there is no preferential observer associated with a photon, it is necessary to express the distribution in terms of observations made by an arbitrary observer, and it is convenient to do this in such a way that the distribution function so introduced is independent of the particular observer chosen. Consider therefore at an event $P(x)$ an observer $A$ whose world-line is tangent to the vector $h^{i}$ at $P$, and let $d V$ be the element of volume of $A$ 's instantaneous space, i.e. the space orthogonal to $h^{i}$. Also let $d \omega$ be the element of twodimensional solid angle, measured by $A$, which is defined by the range $\left(p^{i}, p^{i}+d p^{i}\right)$ at $P$; and if $E$ is the energy of the photon $p^{i}$ measured by $A$, let $(E, E+d E)$ be the range of $E$ defined by the range $\left(p^{i}, p^{i}+d p^{i}\right)$. Then it can easily be verified that for a given distribution at $P$, the expressions $E d V, E d E d \omega$ are separately invariant for a change of observer.

If $d N$ is the number of photons crossing $d V$ and issuing inside the solid angle $d \omega$ and with energies in the range $(E, E+d E)$, we can express this number in the form

$$
d N=c^{-6} \psi E^{2} d E d V d \omega,
$$

the factor $c^{-6}$ being included for convenience. Then since $E d V$, $E d E d \omega$ are invariant, we see at once that $\psi$ is also invariant for a change of observer, i.e. $\psi$ is a function of $(x)$ and $(p)$ and does not involve $h^{i}$. Writing

we thus have

$$
d \Omega_{2}=c^{-4} E d E d \omega
$$

$$
d N=c^{-2} \psi(x, p) E d V d \Omega_{2} .
$$

Assuming that the coordinate system is chosen as in $\S 3$, we can choose the observer making the counts to be an observer whose world-line is orthogonal to the surface $x^{0}=$ constant at $P(x)$. Then from (30) and (1), we find

$$
E=c^{2} p^{0}\left(g^{00}\right)^{-1}, \quad d V=\left(-g g^{00}\right)^{\frac{1}{2}} d x^{1} d x^{2} d x^{3} .
$$

If also we allow $p^{1}, p^{2}, p^{3}$ to be the independent components of the vector $p^{i}$, then it can easily be verified that ${ }^{1}$

$$
d \Omega_{2}=(-g)^{\natural}\left(p_{0}\right)^{-1} d p^{1} d p^{2} d p^{3} .
$$

${ }^{1}$ This expression for $d \Omega_{2}$ may be compared with the expression for $d \Omega_{1}$ given by (23). 
The components $p^{0}, p_{0}$ must now be expressed in terms of $p^{\mu}$ by means of $(28)$ and $p_{0}=g_{0 i} p^{i}$. In order that the vector $p^{i}$ shall have the correct sense, $p^{0}$ must be the positive root of the equation (28). It is seen that the components $p^{\mu}$ are not restricted, and the corresponding value of $p^{0}$ is unique. Substituting in (32), we now have

$$
d N=\psi(x, p)(-g)\left(p_{0}\right)^{-1} p^{0} d x^{1} d x^{2} d x^{3} d p^{1} d p^{2} d p^{3} .
$$

It is required that the number of photons shall be conserved, whence the distribution function $\psi(x, p)$ must be such that

$$
\Delta(d N)=0,
$$

where $\Delta$ denotes the increment for a displacement from $P(x)$ along the path in direction $p^{i}$. Passing along the path in the direction $p^{i}$ from the surface $x^{0}$ to the surface $x^{0}+\Delta x^{0}$, where $\Delta x^{0}$ is small and positive, and writing $x^{i}=x^{i}+\Delta x^{i}, p^{i}=p^{i}+\Delta p^{i}$, we get from (29),

where

$$
\begin{aligned}
x^{i} & =x^{i}+\epsilon p^{i}, \\
p^{i} & =p^{i}-\epsilon \Gamma_{j k}^{i} p^{i} p^{k}, \\
\epsilon & =\Delta x^{0} / p^{0} .
\end{aligned}
$$

Calculating $\partial\left(x^{\prime 1}, \ldots, p^{\prime 1}, \ldots\right) / \partial\left(x^{1}, \ldots, p^{1}, \ldots\right)$ from these relations, and remembering that $p^{0}$ is given by (28), we find that

$\Delta\left(d x^{1} . d p^{1} ..\right)=\epsilon\left\{-2 \Gamma_{i k}^{i} p^{k}+\left(p^{0}\right)^{-1} \Gamma_{j k}^{0} p^{j} p^{k}+\left(p_{0}^{k}\right)^{-1} \Gamma_{0 k}^{i} p_{i} p^{k}\right\} d x^{1} . . d p^{1} .$. Also,

Hence

$$
\begin{aligned}
& \Delta(-g)=-\epsilon 2 g \Gamma_{i k}^{i} p^{k}, \\
& \Delta\left(p^{0}\right)=-\epsilon \Gamma_{j k}^{0} p^{j} p^{k}, \\
& \Delta\left(p_{0}\right)=\epsilon \Gamma_{0 k}^{i} p_{i} p^{k}
\end{aligned}
$$

$$
\Delta\left\{-g\left(p_{0}\right)^{-1} p^{0} d x^{1} \ldots d p^{1} . .\right\}=0 .
$$

Thus, from (34), equation (35) becomes $\Delta \psi=0$, i.e.

$$
\frac{\partial \psi}{\partial x^{i}} p^{i}-\frac{\partial \psi}{\partial p^{i}} \Gamma_{j k}^{i} p^{j} p^{k}=0
$$

This therefore is the generalised Boltzmann equation that must be satisfied by the distribution function $\psi(x, p)$ in order that the number of photons shall be conserved.

$\S 8$. The energy tensor for the system of photons.

The energy tensor for a system of photons is defined to be

$$
T_{2}^{i j}=\int p^{i} p^{j} c^{2} E^{-1} d \sigma
$$

where $d \sigma$ is the number-density relative to some observer and $E$ is the energy relative to this observer of the photon with momentum 
vector $p^{i}$, the integration being over all energies and directions. The expression (18) for $T_{1}^{i j}$ is equivalent to (37), for then $d \sigma$ is the proper density, so that $E=m_{0} c^{2}$. From (32), the number-density is

whence

$$
d \sigma=\frac{d N}{d \bar{V}}=c^{-2} \psi(x, p) E d \Omega_{2},
$$

$$
T_{2}^{i j}=\int \psi(x, p) p^{i} p^{j} d \Omega_{2} .
$$

In the coordinate system referred to above, $d \Omega_{2}$ is given by (33), and since the components $p^{\mu}$ are not restricted, the limits of integration in $(38)$ are $(-\infty,+\infty)$ for each variable.

Proceeding exactly as in $\S 5$, we find

whence

$$
T_{2, k}^{i j}=\int\left(\frac{\partial \psi}{\partial x^{k}}-\frac{\partial \psi}{\partial x^{l}} \Gamma_{h k}^{l} p^{h}\right) p^{i} p^{j} d \Omega_{2},
$$

$$
T_{2}^{i j},{ }_{j}=0
$$

when $\psi(x, p)$ satisfies equation (36). Hence, the energy tensor for $a$ conserved system of photons satisfies the equations $T^{i j},{ }_{j}=0$.

\section{§9. The complete energy tensor.}

Combining the systems of material particles and photons, the complete energy tensor is

$$
T^{i j}=T_{1}^{i j}+T_{2}^{i j}
$$

Thus the energy tensor is constructed from functions $\psi(x, \lambda), \psi(x, p)$ satisfying the Boltzmann equations (21) and (36).

From (27) and (39), we see that the energy tensor satisfies the equations of conservation

$$
T^{i j},{ }_{j}=0 .
$$

$\S 10$. The converse problem.

The converse problem is:-given a tensor $T^{i j}$ satisfying (41), what functions $\phi, \psi$, if they exist, are such that they satisfy (21) and (36), and such that $T^{i j}$ can be derived from these functions by the above process? No solution of this problem has yet been found, but there is little doubt that a solution exists and that it is not unique.

In the general theory of relativity, the energy tensor is related to the form of space-time by the field equations, which can be written in the form

$$
R^{i j}-\Lambda g^{i j}=\kappa\left(\frac{1}{2} T g^{i j}-T^{i j}\right), \quad T=g_{i j} T^{i j},
$$


where $R_{i j}$ is the contracted curvature tensor, $R=g^{i j} R_{i j}, \kappa$ is Einstein's constant, and $\Lambda$ is the cosmical constant. The problem now becomes essentially geometrical: what functions $\phi, \psi$, if they exist, will lead to equations (42)? We have

$$
T^{i j}=\int \phi \lambda^{i} \lambda^{j} d \Omega_{1}+\int \psi p^{i} p^{j} d \Omega_{2},
$$

and since $g_{i j} \lambda^{i} \lambda^{j}=1, g_{i j} p p^{j}=0$,

$$
T=\int \phi d \Omega_{1}
$$

Substituting in (42), functions $\phi, \psi$, satisfying (21) and (36), are required so that the integral equations

$$
R^{i j}-\Lambda g^{i j}=\kappa \int \phi\left(\frac{1}{2} g^{i j}-\lambda^{i} \lambda^{j}\right) d \Omega_{1}-\kappa \int \psi p^{i} p^{j} d \Omega_{2}
$$

shall be satisfied at all points of space-time. This problem has not yet been solved, and a solution would be interesting from the point of view of geometry as well as physics.

\section{§11. The Lemaître universe.}

For a space-time of the form studied by Lemaitre and other writers, equations (21) and (36) can be solved for $\phi$ and $\psi$, and the integral equations (43) become more explicit. The metric is now

$$
d s^{2}=c^{2} d t^{2}-R^{2} h_{\mu^{\prime}} d x^{\mu} d x^{\nu},
$$

where $R$ is a function ${ }^{1}$ of $t$, the $h$ 's are independent of $t$, and $h_{\mu \nu} d x^{\mu} d x^{\nu}$ is the metric of a three-space of constant curvature 1,0 , or -1 . Thus the coordinate $t$ plays the part of $x^{0}$ and has the properties stated in $\S 3$.

It is evident from symmetry that $\phi$ is required to involve only $t$ and $\lambda^{0}(=d t / d s)$. Calculating the Christoffel symbols, equation (21) becomes

$$
\frac{\partial \phi}{\partial t} \lambda^{0}-\frac{\partial \phi}{\partial \lambda^{0}} \frac{1}{c^{2}} R R^{\prime} h_{\mu \nu} \lambda^{\mu} \lambda^{\prime \prime}=0, \quad R^{\prime}=\frac{d R}{d t},
$$

i.e., since $R^{2} h_{\mu \nu} \lambda^{\mu} \lambda^{\nu}=c^{2}\left(\lambda^{0}\right)^{2}-1$ from (44),

$$
\frac{c^{2} \lambda^{0}}{c^{2}\left(\lambda^{0}\right)^{2}-1} \frac{\partial \phi}{\partial t}-\frac{R^{\prime}}{R} \frac{\partial \phi}{\partial \lambda^{0}}=0
$$

1 This function $R(t)$ must not be confused with the scalar curvature $R=g^{i j} R_{i j}$. 
This equation can at once be integrated to give

$$
\phi=f\left[R \sqrt{ }\left\{c^{2}\left(\lambda^{0}\right)^{2}-1\right\}\right]
$$

where $f$ is an arbitrary function of its argument.

From (44), $(-g)^{\frac{h}{2}}=\left.c R^{3}{ }_{\mid} h_{\mu \nu}\right|^{\hat{1}}=c R^{3} h^{\mathrm{h}}$, and $\lambda_{0}=c^{2} \lambda^{0}$. The energy tensor $T_{1}^{i j}$ is therefore, from (24),

$$
T_{1}^{i j}=\iiint_{-\infty}^{+\infty} f \lambda^{i} \lambda^{j}\left(c \lambda^{0}\right)^{-1} R^{3} h^{\underline{1}} d \lambda^{1} d \lambda^{2} d \lambda^{3},
$$

where $c \lambda^{0}=\left(1+R^{2} h_{\mu \nu} \lambda^{\mu} \lambda^{\nu}\right)^{\frac{1}{1}}$. These integrals can be evaluated without much difficulty, and we find, on writing $R^{2} h_{\mu \nu} \lambda^{\mu} \lambda^{\nu}=r^{2}$,

$$
\begin{aligned}
& T_{1}^{00}=\frac{4 \pi}{c^{2}} \int_{0}^{\infty} f(R r)\left(1+r^{2}\right)^{\frac{1}{2}} r^{2} d r, \\
& T_{1}^{0 \mu}=0, \\
& T_{1}^{\mu_{\nu}}=-g^{\mu \nu} \frac{4 \pi}{3} \int_{0}^{\infty} f(R r)\left(1+r^{2}\right)^{-\frac{1}{2}} r^{4} d r .
\end{aligned}
$$

Writing for convenience

$$
F(x)=\frac{4}{3} \pi \kappa x^{2} f(x),
$$

where $\kappa$ is Einstein's constant, these expressions become

$$
\begin{aligned}
\kappa T_{1}^{00} & =\frac{3}{c^{2} R^{2}} \int_{0}^{\infty} F(R r)\left(1+r^{2}\right)^{\sharp} d r, \\
T_{1}^{0 \mu} & =0, \\
\kappa T_{1}^{\mu \nu} & =-g^{\mu \nu} \frac{1}{R^{2}} \int_{0}^{\infty} F(R r)\left(1+r^{2}\right)^{-\frac{1}{2}} r^{2} d r .
\end{aligned}
$$

The procedure for finding $\psi$ and $T_{2}^{i j}$ is very similar. Assuming that $\psi$ involves only $t$ and $p^{0}$, equation (36) gives

$$
\psi=g\left(c R p^{0}\right)
$$

where $g$ is an arbitrary function of its argument. Writing $R^{2} h_{\mu \nu} p^{\mu} p^{\nu}=r^{2}$, we find from (38) and (33),

Writing

$$
\begin{aligned}
& T_{2}^{00}=\frac{4 \pi}{c^{2}} \int_{0}^{\infty} g(R r) r^{3} d r, \\
& T_{2}^{0 \mu}=0 \\
& T_{2}^{\mu \nu}=-g^{\mu \nu} \frac{4 \pi}{3} \int_{0}^{\infty} g(R r) r^{3} d r .
\end{aligned}
$$

$$
K=\frac{4}{3} \pi \kappa \int_{0}^{\infty} g(x) x^{3} d x
$$


we have

$$
\begin{aligned}
\kappa T_{2}^{00} & =\frac{3 K}{c^{2} R^{4}}, \\
T_{2}^{0 \mu} & =0 \\
\kappa T_{2}^{\mu \nu} & =-\frac{K}{R^{4}} g^{\mu \nu} .
\end{aligned}
$$

Thus, finally, from (40),

$$
\begin{aligned}
\kappa T^{00} & =\frac{3 K}{c^{2} R^{4}}+\frac{3}{c^{2} R^{2}} \int_{0}^{\infty} F(R r)\left(1+r^{2}\right)^{\frac{1}{2}} d r, \\
T^{0 \mu} & =0, \\
\kappa T^{\mu \nu} & =-g^{\mu \nu}\left\{\frac{K}{R^{4}}+\frac{1}{R^{2}} \int_{0}^{\infty} F(R r)\left(1+r^{2}\right)^{-\frac{1}{3}} r^{2} d r\right\} .
\end{aligned}
$$

Adopting the value $\delta \pi \gamma / c^{2}$ for $\kappa$, the relative density, $\rho_{00}$, and the pressure, $p$, are given by

$$
T^{i j}=\left(\rho_{00}+\frac{p}{c^{2}}\right) \frac{1}{c^{2}} \delta_{0}^{i} \delta_{0}^{j}-\frac{p}{c^{2}} g^{i j},
$$

whence, from (46) and (48),

$$
\begin{aligned}
& \kappa \rho_{00}=\frac{3 K}{R^{4}}+\frac{3}{R^{2}} \int_{0}^{\infty} F(R r)\left(1+r^{2}\right) \frac{1}{d} d r \\
& \frac{\kappa}{c^{2}} p=\frac{K}{R^{4}}+\frac{1}{R^{2}} \int_{0}^{\infty} F(R r)\left(1+r^{2}\right)^{-\frac{1}{2}} r^{2} d r .
\end{aligned}
$$

Hence, the proper density, $\rho_{0}=\rho_{00}-3 p / c^{2}$, is given by

$$
\kappa \rho_{0}=\frac{3}{R^{2}} \int_{0}^{\infty} F(R r)\left(1+r^{2}\right)^{-\frac{1}{2}} d r .
$$

It can easily be verified from (50) and (51) that

$$
\frac{d}{d t}\left(R^{3} \rho_{00}\right)+\frac{p}{c^{2}} \frac{d}{d t}\left(R^{3}\right)=0 .
$$

This is to be expected, for the relation (53) can be deduced immediately from (41), (44) and (49).

Expressions for $\rho_{00}$ and $p$ in terms of $R(t)$ are obtained from the field equations (42). We need not however consider both expressions, for $p$ is given by (53) when $\rho_{00}$ is known. Calculating $\rho_{00}$ from (49), (42) and (44), we find

$$
\kappa \rho_{00}=\frac{3 R^{\prime 2}}{c^{2} R^{2}}+\frac{3 k}{R^{2}}-\Lambda
$$


where $k(=1,0$, or -1$)$ is the constant curvature of the form $h_{\mu \nu} d x^{\mu} d x^{\nu}$ in (44). Hence, from (50) and (45), the integral equations (43) reduce to the equations

$$
\begin{aligned}
\int_{0}^{\infty} g(x) x^{3} d x & =\frac{3 K}{4 \pi \kappa}, \\
\int_{0}^{\infty} F(R r)\left(1+r^{2}\right)^{\frac{1}{b}} d r & =\frac{1}{c^{2}} R^{\prime 2}+k-\frac{1}{3} \Lambda R^{2}-\frac{K}{R^{2}} .
\end{aligned}
$$

Here $K$ can be considered as an arbitrary constant provided the right-hand side of (55) is always positive.

When $R$ is a given function of $t, R^{\prime}$ can be expressed in terms of $R$, and (55) is an integral equation for the function $F$, the parameter being $R$. Conversely, when the functions $F, g$ are given, equations (54) and (55) can be used to find the form of $R$ as a function of $t$. Thus the form $R(t)$ can be found if the distribution at any one event is known provided the value of $R$ is known at that event. 